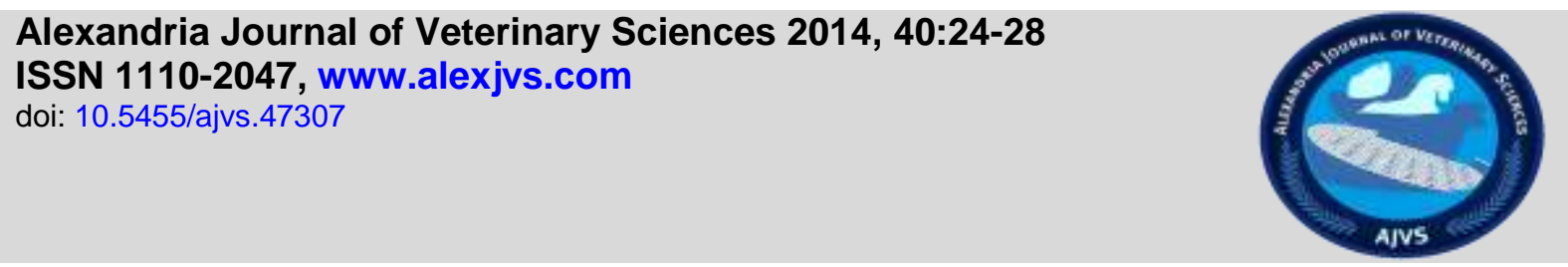

\title{
Evaluation of a Rapid Immunochromatographic Test for Detection of Brucella Abortus Antibodies in Egyptian Cattle Sera and Milk
}

\author{
Tharwat M. Elshemey and Amir H. Abd-Elrahman \\ Department of Animal Medicine, Faculty of Veterinary Medicine, Alexandria University.
}

\begin{tabular}{ll}
\hline Key words: & ABSTRACT: \\
Brucella & Rapid diagnostic tests are needed to facilitate diagnosis and control of \\
abortus, & brucellosis. Sero-epidemiology of brucellosis is currently done by employing \\
RBT, & the Rose Bengal test (RBT). Also the world organization for animal health \\
Immunochrom & (OIE) has approved an indirect ELISA for testing serum and milk. The \\
atographic & Immuno-Chromatographic brucellosis test (ICT) is a rapid, card-based \\
test, & immunochromatographic test for detection of antibodies directed against $B$. \\
ELISA. & abortus antigens. To the best of our knowledge, ICT has not been used for the \\
& diagnosis of brucellosis in cattle yet. The objective of the study was to evaluate \\
& the performance of the ICT brucellosis test for the diagnosis of $B$. abortus in \\
& cattle sera versus RBT and ELISA as a gold standard, Also evaluation of ICT \\
& brucellosis test efficacy for detection of antibodies against $B$. Abortus in milk \\
& samples. $94,90.36$ and $84.3 \%$ of sera samples were positive by RBT, ICT and \\
& ELISA respectively. The ICT had $94.44 \%$ sensitivity and $100 \%$ specificity \\
& versus RBT and ELISA as a gold standard. The kappa value between ICT and \\
& ELISA was 0.76. Also ICT detected antibodies against $B$. abortus in serum and \\
milk samples of 7 cows while a recently calved cow showed antibodies in the \\
milk sample only. In conclusion, ICT provides a handy and accurate tool for \\
the rapid detection of antibodies to $B$. abortus in cattle sera and milk.
\end{tabular}

Corresponding Author: Amir H. Abd-Elrahman, amirhamed22@yahoo.com

\section{INTRODUCTION}

Brucellosis is a worldwide zoonosis caused by the facultative intracellular members of the bacterial genus Brucella of which at least six species are now recognized; these are highly genetically homogenous, and the disease is of major socio-economic importance, Commander et al., (2002). The disease still constitutes a great threatening

challenge to livestock breeding all over the world despite being successfully eradicated from many developed countries. Eradication programs in these countries depended largely on testing and slaughtering of positive animals, (Robinson, 2003). In developing countries, use of conventional routine methods of diagnosis which lack high accuracy, Rajaii et al., (2006), and the poor public knowledge of the disease greatly tie up the development of successful eradication programs (Bekele et al., 2011).

Classical tests for the diagnosis of brucellosis i.e. culture and phenotypic characterization, are laborious, time-consuming, pose the risk of infection, and can generate discordant results (Muma et al., 2009). Serological tests are therefore commonly used for Brucella diagnosis in cattle and small ruminants especially at herd level. Rose Bengal Test (RBT), Complement Fixation Test (CFT) and ELISA (Enzyme Linked Immuno Sorbent Assay) are widely used for the detection of antibodies to Brucella spp. The sensitivity of RBT fulfills the requirements for surveillance of free areas at flock level but it is believed 
that only the combination of RBT and CFT or ELISA in infected flocks can obtain accurate individual sensitivity in test-and-slaughter programs (Munoz et al., 2005).

The ICT (Immunechromatographic Test) is a simple and rapid method, which makes it suitable for clinical and field applications. It relies on migration of liquid across the surface of a nitrocellulose membrane and because of its convenience has been developed for a variety of applications over the past decade. Assays using this format take approximately 15 minutes to complete and require only a small volume $(<200 \mu \mathrm{L})$ of the tested serum or milk on the test strip (Chandler et al., 2000).

The aim of this study was to evaluate the clinical utility of the ICT as a rapid method for the detection of brucella abortus antibodies in cattle sera compared to RBT and ELISA, Also evaluation of ICT brucellosis test efficacy for detection of antibodies against B. abortus in milk samples.

\section{MATERIALS AND METHODS}

\subsection{Samples:}

Eighty three sera samples were collected from cows belong to a dairy herd with a history of abortions, retained placenta, stillbirths and fertility problems. Sera were tested with RBT, ICT and indirect ELISA. "Also" 11 sera and milk samples collected from other cows from the same herd were tested using rapid ICT for detection of antibodies against brucellosis.

\subsection{Rapid immune-chromatographic test:}

Anigen rapid $B$. Brucella Ab test kit (one step bovine brucella antibody rapid test) was obtained from BIONOTE, 2-9, Seogu-dong, Hwaseong-si, Gyeonggi-do, Korea. The test was applied on sera and milk samples according to enclosed leaflet.

\subsection{Rose Bengal plate test (RBT):}

Antigen for RBT was obtained from Veterinary Serum and Vaccine Research Institute, Abbasia, Cairo, Egypt. The RBT was performed according to standard procedures (Alton et al., 1988).

\subsection{ELISA:}

Antibodies in sera from cattle and calves were investigated by indirect ELISA (Anigen B. Brucella Ab ELISA) which was obtained from BIONOTE, 2-9, Seogu-dong, Hwaseong-si, Gyeonggi-do, Korea. The test was applied according enclosed leaflet.

\subsection{Sensitivity and specificity:}

RBT and indirect ELISA were used as a "gold standard" to estimate the sensitivity and specificity of the ICT.

\subsection{Statistical analysis.}

The strength of agreement between the ICT, RBT and ELISA was estimated by calculating the kappa statistic. Kappa statistic values $>$ $0.75, \quad 0.40-0.75$, and $<0.40$ represent excellent agreement, good to fair agreement, and poor agreement, respectively according to (Sim and Wright, 2005).

\section{RESULTS}

3.1. Detection of brucella abortus antibodies in Egyptian cattle serum:

A total of 83 samples of cattle sera were tested by using RBT, ICT and ELISA from a dairy herd with a history of abortion, retained placentas, stillbirths and infertility problem. The results revealed that $94,84.3$ and $90.36 \%$ were positive samples by using RBT, ICT and ELISA respectively as shown in Table 1.

\subsection{Sensitivity and specificity of rapid test for detection of brucella abortus antibodies in sera of examined cattle as compared to RBT \& ELISA:}

Table (2): ICT showed $94.44 \%$ sensitivity and $100 \%$ specificity versus RBT and ELISA as a gold standard.

\subsection{Efficacy of ICT for detection of brucella abortus antibodies in milk \\ Antibodies against $B$. abortus was detected in serum and milk samples of 7 cows while a recently calved cow showed antibodies in the milk sample only.}


Table (1) Comparison of three methods used for detection of brucella abortus antibodies in examined cattle sera.

\begin{tabular}{cccc}
\hline Number of samples & RBT & ICT & ELISA \\
\hline 68 & + & + & + \\
1 & - & + & + \\
2 & - & - & + \\
1 & + & + & - \\
5 & + & - & - \\
4 & + & - & - \\
2 & - & $70(84.3 \%)$ & $75(90.36 \%)$ \\
\hline Total $=83$ & $78(94 \%)$ & & \\
\hline
\end{tabular}

Table (2) Sensitivity and specificity of rapid test for detection of brucella abortus antibodies in sera of examined cattle as compared to RBT and ELISA

\begin{tabular}{ccccc}
\hline & True positive & True negative & Sensitivity (\%) & Specificity (\%) \\
\hline RBT\&ELISA & 72 & 2 & 100 & 100 \\
ICT & 68 & 2 & 94.44 & 100 \\
\hline
\end{tabular}

True positive means that animal was positive with RBT and ELISA together.

True negative means that animal was negative with RBT and ELISA together.

Sensitivity means (number of samples in which the test was positive when RBT and ELISA were positive/ True positives X 100).

Specificity means (number of samples in which the test was negative when RBT and ELISA were negative/ number of true negatives $X 100$ ).

\section{DISCUSSION}

Bovine brucellosis caused by B. abortus, characterized by a storm of abortion in free herds followed by reproductive problems and sporadic cases of abortion in the subsequent seasons while only sporadic cases of abortion are experienced when the disease is caused by $B$. melitensis because cattle is not the preference host of the later organism (Hussein, 2005).

Control of brucellosis in livestock depends on the reliability of the methods used for detection and identification of the causative agent, also rapid and accurate methods are needed. The standardization and, to a great extent, the final performance of serological tests rely on the definitions of their sensitivity and specificity with sets of sera representative of the context in which they are to be applied (Taddepalli et al., 2011). In this work, the positive control sera were selected on the basis of a positive serological result in the standard Rose Bengal and

\section{ELISA tests.}

The obtained results revealed that $94,84.3$ and $90.36 \%$ of the cattle sera investigated were positive by RBT, ICT and ELISA, respectively (Table 1). RBT detected more positive cases than all other serological tests used (94\%). Thus, RBT seems to be a valuable tool for the diagnosis of brucellosis in cattle especially when taking into consideration the speed, the objectivity of result interpretation and the cost factors. Various studies, however, have confirmed that ELISA techniques perform better than other conventional tests used for serological screening of brucellosis in cattle (Gwida et al., 2011).

Our results revealed that 70 cattle serum samples out of $83(84.3 \%)$ were positive by ICT, demonstrating moderate sensitivity when compared to other serological tests. This may be attributed to the fact that sensitivity and specificity may vary considerably if another gold standard used as 
ELISA test usually reacts nonspecifically with other similar antigens (Rajaii et al., 2006). To the best of our knowledge, ICT has not been used for the diagnosis of brucellosis in cattle yet, so this is the first report discussing the application of this test for detection of brucellosis antibodies in cattle sera and milk, however only one report evaluated the clinical utility of ICT for serodiagnosis of dogs suspected of having brucellosis (Kim et al., 2007).

The obtained results showed that sensitivity and specificity of ICT were 94.44 and 100\% respectively as compared to RBT and ELISA as a gold standard (Table 2). These results showed that immunochromatography test strips are technically fully developed and that they exhibit a series of specific advantages as investigations can be performed rapidly and simply, without requiring special instruments or expertise in the method. As the sensitivity and specificity of many test procedures are now really high, rapid tests can be extraordinarily useful in answering specific questions and thus in helping to orientate diagnosis, uncovering chains of infection, and in deciding to start early interference (Stürenburg and Junker, 2009).

A perfect agreement between ICT, RBT and ELISA was proven by calculating Kappa values (0.76) but its sensitivity is lower. It is advisable to combine at least two serological test methods to screen brucellosis on the herd level. Thus, the sensitivity will be increased. Nevertheless, serological methods used solitary or in combination carry the risk to miss seronegative carriers of Brucella (Lorraine et al., 2005).

In this study, ICT detected antibodies against $B$. abortus in serum and milk samples of 7 cows while a recently calved cow showed antibodies in milk sample only, these results showed that ICT could be used perfectly to detect antibodies in milk as well as serum. The obtained result suggests that ICT can be applied on milk of recently calved cows for detection of $B$. abortus antibodies providing earlier diagnosis of $B$. abortus infection than serum testing to avoid negative serum phenomenon in recently calved cows (Mahmoud, 2012).

\section{Conclusion:}

The obtained results in the current study suggest that the ICT is a simple and rapid method that provides accurate detection of antibodies to $B$. abortus in cattle sera and milk samples, thereby saving time and eliminating the need for special training. This rapid test can therefore be practically implemented in epidemiologic surveys for bovine brucellosis, although evaluation on a larger scale with various cattle sera and milk samples is still necessary.

\section{REFERENCES}

Alton, G.G., Jones, L.M., Angus, R.D. Verger, J.M. 1988. Techniques for the brucellosis laboratory. Institut National de la RechercheAgronomique, Paris, France. 81-134.

Bekele, M., Demelash, B.,Fekadu, N., Tesfaye, R., Kassahun, A. Eystein, S. 2011. Cattle brucellosis in traditional livestock husbandry practice in Southern and Eastern Ethiopia, and its zoonotic implication. Acta. Vet. Scand. 53 (1):24-31.

Chandler, J., Gurmin, T. Robinson, N. 2000. "The Place of Gold in Rapid Tests", IVD Technology 6, no. 2: 37-49.

Commander, N.J., Vickers, A., Cutler, S., Wren, B. MacMillan, A.P. 2002. DNA based vaccines for protection of man and animals against brucellosis. 55th Annual Brucellosis Research Conference November 9-10, Millennium Hotel St. Louis, MO.

Gwida, M.M., El-Gohary, A.H., Melzer, F., Tomaso, H., Rosler, U., Wernery, U., Wernery, R., Elschner, M.C, Khan, I., Eickhoff, M., Schoner, D. Neubauer, H. 2011. Comparison of diagnostic tests for the detection of Brucella spp. in camel sera.BMC Res Notes. 6;4:525-531.

Hussein HI. Textbook "Infectious diseases of domestic animals" Lazer center publication and distribution, Egypt, Beni-suef. 2005.

Kim, J.W., Lee, Y.J., Han, M.Y., Bae, D.H., Jung, S.C., Oh, J.S., Ha, G.W., Cho, B.K., 2007. Evaluation of immunochromatographic assay for serodiagnosis of Brucella canis.J Vet Med Sci. 69(11):1103-1107.

Lorraine, L.P., John, A.M., Simon, D.B., Judith, A.S. 2005.Evaluation of Competitive ELISA for Detection of Antibodies to Brucella Infection in Domestic Animals. ClinDiagn Lab Immunol. 12(1): 141-151. 
Mahmoud AA. Textbook "Infectious diseases of domestic animals" Faculty of Veterinary Medicine, Alexandria University.Egypt, 2012.

Muma, J.B., Lund, A., Nielsen, K., Matope, G., Munyeme, M., Mwacalimba, K., Skjerve, E. 2009. Effectiveness of Rose Bengal test and fluorescence polarization assay in the diagnosis of Brucella spp. infections in free range cattle reared in endemic areas in Zambia. Trop Anim Health Prod. 41(5):723-729.

Munoz, P.M., Marin, C.M., Monreal, D., Gonzalez, D., Garin-Bastuji, B., Mainar-Jaime, R.C., Moriyon, I., Blasco, J.M. 2005. Efficacy of Several Serological Tests and Antigens for Diagnosis of Bovine Brucellosis in the Presence of False-Positive Serological Results Due to Yersinia enterocolitica O:9. Clin. Diagn. Lab. Immunol. 12(1): 141-151.

Rajaii, M., Naghili, B. Pourhassan, A. 2006. Comparison of ELISA and STA tests in diagnosis of Brucellosis. Iranian Journal of Clinical Infectious Diseases 1(3):145-147.

Robinson A. (Guidelines for co-ordinated human and animal brucellosis surveillance. FAO Animal production and health paper, 2003, ISSN: 02546019.

Sim, J. Wright, C.C. 2005. "The Kappa Statistic in Reliability Studies: Use, Interpretation, and Sample Size Requirements". Physical Therapy 85: 257-268.

Stürenburg, E. Junker, R. 2009. Point-of-Care Testing in Microbiology-The Advantages and Disadvantages of Immunochromatographic Test Strips. Dtsch. Arztebl. Int. 106(4): 48-54.

Tadepalli, M., Lingala, R., Trangadia, B., Dorairahan, T. Villuppanoor, A.S. 2011. Development and Comparative Evaluation of a Competitive ELISA with Rose Bengal Test and a Commercial Indirect ELISA for Serological Diagnosis of Brucellosis. Indian J Microbiol. 2011 October; 51(4): 528-530. 